\title{
Substance Abuse
}

National Cancer Institute

\section{Source}

National Cancer Institute. Substance Abuse. NCI Thesaurus. Code C18272.

Maladaptive pattern of drug or alcohol use that may lead to social, occupational, psychological, or physical problems. 